\title{
Applying Statistical and Complex Network Methods to Explore the Key Signaling Molecules of Acupuncture Regulating Neuroendocrine-Immune Network
}

\author{
Kuo Zhang, ${ }^{1}$ Xin-meng Guo, ${ }^{2}$ Ya-wen Yan, ${ }^{1}$ Yang-yang Liu $\mathbb{D}^{1,}, 3$ Zhi-fang Xu, ${ }^{1,3}$ \\ Xue Zhao, ${ }^{1,3}$ Jiang Wang, ${ }^{2}$ Yi Guo $\mathbb{D}^{1,4} \mathrm{Kai} \mathrm{Li}^{5}$ and Sha-sha Ding ${ }^{6}$ \\ ${ }^{1}$ Research Center of Experimental Acupuncture Science, Tianjin University of Traditional Chinese Medicine, Tianjin, China \\ ${ }^{2}$ School of Electrical Engineering and Automation, Tianjin University, Tianjin, China \\ ${ }^{3}$ Acu-Moxibustion and Tuina Department, Tianjin University of Traditional Chinese Medicine, Tianjin, China \\ ${ }^{4}$ College of Traditional Chinese Medicine, Tianjin University of Traditional Chinese Medicine, Tianjin, China \\ ${ }^{5}$ Department of Gynaecology, First Teaching Hospital of Tianjin University of Traditional Chinese Medicine, Tianjin, China \\ ${ }^{6}$ Department of Acupuncture and Moxibustion, Tianjin Nankai Hospital, Tianjin, China \\ Correspondence should be addressed to Yang-yang Liu; lyy02211@163.com
}

Received 29 August 2017; Revised 11 December 2017; Accepted 20 December 2017; Published 29 January 2018

Academic Editor: Morry Silberstein

Copyright (C) 2018 Kuo Zhang et al. This is an open access article distributed under the Creative Commons Attribution License, which permits unrestricted use, distribution, and reproduction in any medium, provided the original work is properly cited.

\begin{abstract}
The mechanisms of acupuncture are still unclear. In order to reveal the regulatory effect of manual acupuncture (MA) on the neuroendocrine-immune (NEI) network and identify the key signaling molecules during MA modulating NEI network, we used a rat complete Freund's adjuvant (CFA) model to observe the analgesic and anti-inflammatory effect of MA, and, what is more, we used statistical and complex network methods to analyze the data about the expression of 55 common signaling molecules of NEI network in ST36 (Zusanli) acupoint, and serum and hind foot pad tissue. The results indicate that MA had significant analgesic, anti-inflammatory effects on CFA rats; the key signaling molecules may play a key role during MA regulating NEI network, but further research is needed.
\end{abstract}

\section{Introduction}

Acupuncture is a physical therapy of preventing or treating diseases by inserting needle into specific acupoints. As a kind of nonspecific physical stimulus, the effects of acupuncture are mediated by the regulatory systems in the body. This determines that the basic way of acupuncture effect is regulating the body's condition, with the characteristics of whole regulation.

Researches have shown that the body's inherent regulatory system is neuroendocrine-immune (NEI) network, including nervous system, endocrine system, and immune system, which is the biological basis to maintain the body's homeostasis. The three systems share common signaling molecules and their affiliated receptors, including some neuropeptides, neurotransmitters, cytokines, and hormones, and their receptors [1]. The cells in each system can secrete these signal molecules and at the same time the cells' surface has the moleculars' receptors. Hence, the common signaling molecules and their receptors constitute the molecular structural foundation of NEI network, being responsible for information communication and transmission between the three systems $[1,2]$.

Some researches had demonstrated that acupuncture could regulate one system of the NEI network [3-6], in which most focus on the nervous system or immune system, but little studies pay attention to the regulatory effect of acupuncture on the whole NEI network [7]. Therefore, in order to reveal the regulatory effect of acupuncture on NEI network, in this study, we analyzed 55 common signaling molecules of NEI network in serum, supernatants from the ST36 acupoint, and hind foot pad tissue in rats with inflammatory pain 
after acupuncture treatment and further explore the possible key signaling molecules during acupuncture modulating the NEI network by statistical method. Moreover, in order to characterize the interaction between the common signaling molecules and identify the signaling molecules which play a major role (key signaling molecules) in the NEI network, we applied complex network method for further analysis, which is a powerful tool to solve such network problems $[8,9]$, and hope to provide ideas and methods for fully revealing the mechanism of acupuncture.

\section{Materials and Methods}

2.1. Animal Preparation. The experiments were performed on male Wistar rats (weight: $180 \pm 20 \mathrm{~g}$ ) obtained from the Institute of Hygiene and Environmental Medicine, Academy of Military Medical Sciences, PLA (License number SCXK (army) 2009-003). Rats were housed in animal cages under a 12-h light/dark cycle with food and water available ad libitum for 1 week. All animal procedures in this study were performed according to the International Guide for the Care and Use of Laboratory Animals and were approved by the Animal Ethics Committee of Tianjin University of Traditional Chinese Medicine in China (TCMLAEC2012010).

2.2. Experimental Design. The rats were randomly divided into the following groups: (1) normal saline (NS) group: with normal saline injection, (2) CFA group: with CFA injection, and (3) CFA + manual acupuncture (MA) group: with CFA injection and MA manipulation.

2.3. Inflammatory Pain Model. Rats per group were injected with either $0.1 \mathrm{~mL}$ CFA (Sigma, USA) or NS in the plantar surface of the right hind paw to induce intraplantar inflammation [10].

2.4. Measurement of Thermal Hyperalgesia. Thermal hyperalgesia was assessed by hind paw withdrawal latency (PWL) to a noxious thermal stimulus using a plantar tester (BME-410C, Institute of Biological Medicine, Academy of Medical Science, China). Briefly, rats were placed in a clear plastic chamber $(220 \mathrm{~mm} * 110 \mathrm{~mm} * 280 \mathrm{~mm})$ and allowed to acclimatize for $30 \mathrm{~min}$. A radiant heat stimulus was positioned under the glass floor directly beneath the right hind paw. When the rat withdrew its hind paw, we pressed the button to stop the heat stimulus, and the time was recorded as thermal PWL. Screening pain threshold before experiment: the rats with the PWL higher than $20 \mathrm{~s}$ or lower than $14 \mathrm{~s}$ were excluded from the experiment. A $30 \mathrm{~s}$ cut-off was used to prevent tissue injury. PWL was established by averaging the latency of 3 tests with a $5 \mathrm{~min}$ interval between each test. PWL was measured pre-CFA/normal saline injection and at D0 (after CFA injection), D1 (after MA), D7 (after MA), and D21 (after MA) at 14 o'clock to 16 o'clock.

2.5. Measurement of Hind Paw Swelling. The swelling of rat's right hind paw was measured by volumetric method [11] with a self-made foot volume meter. The measured time was the same as PWL. The hind paw was immersed in a chamber containing PBS up to the boundary between hairy and nonhairy skin. The volume displacement represented the hind paw swelling and was determined by two observers. Paw volume was measured twice before CFA or NS injection (as basal paw volume) and at days 1, 7, and 21 after CFA or NS injection.

2.6. MA Treatment. Rats were immobilized in a holder and acupuncture needles $(0.35 \mathrm{~mm}$ in diameter and $25 \mathrm{~mm}$ in length) were inserted to a depth of 5-7 mm at bilateral ST36 (Zusanli) acupoints. The needles were turned at a rate of 3 spins per second bidirectionally ( 1 spin consisted of clockwise rotation of $180^{\circ}$ and a counterclockwise rotation of $180^{\circ}$ ) for $2 \mathrm{~min}$ at Deqi, mild reinforcing and attenuating. The needles were manipulated every $5 \mathrm{~min}$ for a $30 \mathrm{~min}$ session. The manipulations were performed by the same person at 13 oclock to 14 o'clock using metronome to keep the rhythm. MA treatment was given once a day for 7 consecutive days (day 1-day 7 after CFA injection) and then given every other day (day 8-day 21 after CFA injection), for a total of 14 sessions. In order to ensure the stability and repeatability of the manipulations, the operator practiced manipulation repeatedly at the ATP-II acupuncture manipulation parameter tester (which was manufactured by Shanghai University of Traditional Chinese Medicine Shang Xin Medical Technology Company) before and during the experiment. NS group and CFA group underwent grasping and fixation similar to CFA + MA group.

2.7. Sample Collection. The hair on the right legs was removed from the skin using electronic hair clipper at 1 day before sample collection. After PWL measurement at D21 after CFA injection, rats were anesthetized with chloral hydrate (35 mg/kg, i.p.). Then the blood, local tissues in right ST36, and right hind footpad tissue were collected. The blood samples by abdominal aortic method was placed for $2 \mathrm{~h}$ under room temperature and then were centrifuged for $2000 \mathrm{rpm}$ at $4^{\circ} \mathrm{C}$ for $10 \mathrm{~min}$ to get the serum. After blood collection, the tissues located in the right ST36 $(1 \mathrm{~cm}$ in diameter and $0.5 \mathrm{~cm}$ thick, consisting of skin and subcutaneous and muscle tissues) and hind footpad tissue (skin and muscle) were collected immediately with an scapel. Next all tissues were triturated into homogenate with liquid nitrogen. $4^{\circ} \mathrm{C}$ and $2000 \mathrm{r} / \mathrm{min}$ for $10 \mathrm{~min}$ centrifugation was performed to get supernatants. The serum and supernatants were stored at $-80^{\circ} \mathrm{C}$ before detecting the NEI common signaling molecules. The outline of the experimental protocol is summarized in Figure 1.

2.8. Liquid Chip, RIA, and ELISA Detection. 55 NEI common signaling molecules in rats serum, supernatants form the right ST36 acupoint, and hind footpad tissue were detected in this experiment, including 13 neurotransmitters or neuropeptides, 18 endocrine hormones, and 24 cytokines. Due to the limitation of the detection range of current detecting techniques, only one technique cannot detect all signaling 


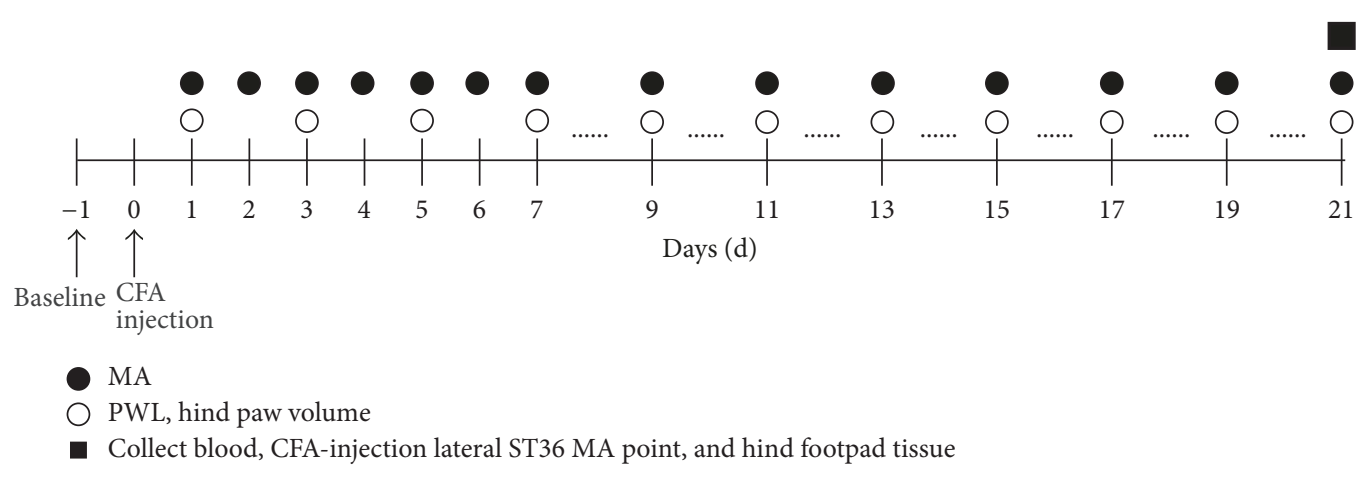

FIGURE 1: Outline of the experimental protocol.

molecules completely, so we combined liquid chip, RIA, and ELISA to detect these signaling molecules.

RIA and ELISA were conducted by Beijing Sinouk Institute of Biological Technology. Rat pituitary, rat stress hormone, rat thyroid, and rat neuropeptide liquid chip kit (Germany, Merck Millipore) were conducted by Beijing Institute of Hepatology. Bio-Plex Pro ${ }^{\mathrm{TM}}$ Rat Cytokine 24-plex Assay liquid chip kit (American, Bio-Rad) was conducted by Beijing Jian Yuan Wei Ye Technology Co., Ltd. Signaling molecules detection was carried out strictly according to the manufacturer's recommendations. The classification and detection methods of the signaling molecules were shown in Table 1.

2.9. Data Analysis. The data about PWL and hind paw swelling were analyzed by statistical analysis method. The data about changes of common signaling molecules in NEI network of 3 kinds of samples induced by MA on day 21 were analyzed by 2 methods, including statistical analysis and complex network analysis.

2.9.1. Statistical Analysis. All statistical tests were conducted using SPSS 19.0 (SPSS Inc, Chicago, IL, USA) software. All statistical data were presented as the mean \pm standard error. A $P$ value $<0.05$ was considered to represent statistical significance.

PWL and hind paw volume data were analyzed using repeated measures analysis of variance (ANOVA), followed by Student-Newman-Keuls test which was used for post hoc analysis for differences between groups. If Mauchly's test of sphericity was not satisfied, One-Way ANOVA followed by LSD or Dunnett's T3 post hoc test were conducted. If data were not normally distributed or violated an assumption of a statistical test, they were transformed using commonly accepted methods or analyzed with a nonparametric test.

Signaling molecules were analyzed using One-Way ANOVA the same as above. The signaling molecules with statistical significance between groups (CFA group compared with NS group or CFA + EA group) were identified as the possible key signaling molecules in MA modulating the NEI network. All figures were generated using GraphPad Prism (GraphPad Software, La Jolla, CA).
2.9.2. Complex Network Analysis. For more direct and visual analysis of the NEI changes induced by MA, all of the 55 common signaling molecules in the samples were analyzed by complex network methods. The complex network analysis method consists of 3 steps. In Step 1, based on the detection results, correlation coefficients between $55 \mathrm{NEI}$ network signaling molecules of the serum, supernatants form the right ST36 point, and hind footpad tissue were calculated by Pearson correlation coefficient formula, and the correlation matrix was constructed by MATLAB software (Natick, America). In Step 2, the thresholds were used to filter the signaling molecules of the correlation coefficients $\in[-1$, $-0.8],[0.8,1]$. In Step 3, screened signaling molecules were sorted by 3 complex network methods (i.e., node strength correlations, node degree, and node clustering coefficient) that could measure the importance of nodes in the network. Two or more methods in The first three nodes obtained by analysis with 2 or 3 methods mentioned above were considered as the key signaling molecules in the NEI network. The 3 complex network methods were as follows. (1) Node strength correlations: in complex network theory, signal molecules were viewed as nodes; the strength correlations were sum of absolute values of correlation coefficients of each node. Nodes were sorted from high to low by strength correlations, a node of higher strength correlations meaning it was more important in the network. (2) Node degree: the degree was the number of edges connected to each node. Nodes were sorted from large to small by degree, a node of larger degree meaning it was more important in the network. (3) Node clustering coefficient: it represented the possibility of connections between the other nodes that were connected to this one node. Nodes were sorted from low to high by the clustering coefficient, a node of lower strength correlations meaning the other nodes are with low possibility of connections except this one node, this also suggested that the node was important in the network.

\section{Results}

3.1. Analgesia Effect of MA on CFA-Induced Hyperalgesia. As shown in Figure 2, there were no differences in PWL under baseline condition (before CFA injection) among the three 
TABLE 1: Classification and detection methods of 55 NEI common signaling molecules.

\begin{tabular}{|c|c|c|c|}
\hline Number & Signaling molecule & Classification & Detection method \\
\hline (1) & Substance P & $\mathrm{N}$ & Liquid chip \\
\hline (2) & 5-Hydroxytryptamine (5-HT) & $\mathrm{N}$ & RIA/ELISA \\
\hline (3) & Calcitonin gene related peptide (CGRP) & $\mathrm{N}$ & RIA/ELISA \\
\hline$(4)$ & Neuropeptide Y (NPY) & $\mathrm{N}$ & RIA/ELISA \\
\hline$(5)$ & Acetylcholine (Ach) & $\mathrm{N}$ & Liquid chip \\
\hline (6) & Vasoactive intestinal peptide (VIP) & $\mathrm{N}$ & Liquid chip \\
\hline (7) & Dopamine (DA) & $\mathrm{N}$ & Liquid chip \\
\hline$(8)$ & Leu-Enkephalin (LEK) & $\mathrm{N}$ & Liquid chip \\
\hline (9) & $\beta$-Endorphin & $\mathrm{N}$ & Liquid chip \\
\hline (10) & Stem cell factor (SCF) & $\mathrm{N}$ & RIA/ELISA \\
\hline (11) & Insulin-like growth factor-1 (IGF-1) & $\mathrm{N}$ & RIA/ELISA \\
\hline (12) & Brain derived neurotrophic factor (BDNF) & $\mathrm{N}$ & Liquid chip \\
\hline (13) & Neurotensin & $\mathrm{N}$ & Liquid chip \\
\hline (14) & Thyroid stimulating hormone (TSH) & $\mathrm{E}$ & Liquid chip \\
\hline (15) & Triiodothyronine (T3) & $\mathrm{E}$ & Liquid chip \\
\hline (16) & Thyroxine (T4) & $\mathrm{E}$ & Liquid chip \\
\hline (17) & Corticotropin releasing hormone $(\mathrm{CRH})$ & $\mathrm{E}$ & RIA/ELISA \\
\hline (18) & Adreno-cortico-tropic-hormone (ACTH) & $\mathrm{E}$ & Liquid chip \\
\hline (19) & Corticosterone & $\mathrm{E}$ & Liquid chip \\
\hline$(20)$ & Follicle-stimulating hormone (FSH) & $\mathrm{E}$ & Liquid chip \\
\hline$(21)$ & Luteinizing hormone (LH) & $\mathrm{E}$ & Liquid chip \\
\hline$(22)$ & Growth-hormone-releasing hormone (GHRH) & $\mathrm{E}$ & RIA/ELISA \\
\hline (23) & Growth hormone $(\mathrm{GH})$ & $\mathrm{E}$ & Liquid chip \\
\hline$(24)$ & Somatostatin (SS) & $\mathrm{E}$ & Liquid chip \\
\hline$(25)$ & $\alpha-\mathrm{MSH}$ & $\mathrm{E}$ & Liquid chip \\
\hline$(26)$ & Melatonin & $\mathrm{E}$ & Liquid chip \\
\hline (27) & Orexin A & $\mathrm{E}$ & Liquid chip \\
\hline$(28)$ & Oxytocin & $\mathrm{E}$ & Liquid chip \\
\hline$(29)$ & Prolactin $(\mathrm{PRL})$ & $\mathrm{E}$ & Liquid chip \\
\hline$(30)$ & Arginine Vasopressin (AVP) & $\mathrm{E}$ & RIA/ ELISA \\
\hline$(31)$ & Erythropoietin (EPO) & $\mathrm{E}$ & Liquid chip \\
\hline (32) & Growth-related oncogene/keratinocyte-derived chemokines (GRO/KC) & $\mathrm{C}$ & Liquid chip \\
\hline$(33)$ & Macrophage inflammatory protein-1 $\alpha($ MIP-1 $\alpha)$ & $\mathrm{C}$ & Liquid chip \\
\hline$(34)$ & 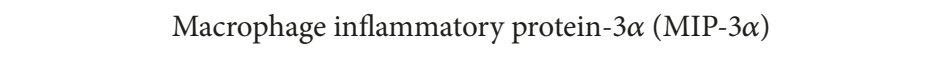 & $\mathrm{C}$ & Liquid chip \\
\hline (35) & Monocyte chemotactic protein 1 (MCP-1) & $\mathrm{C}$ & Liquid chip \\
\hline$(36)$ & Regulated on activation, normal T cell expressed and secreted (RANTES) & $\mathrm{C}$ & Liquid chip \\
\hline (37) & Interleukin 1 alpha (IL-1 $\alpha)$ & $\mathrm{C}$ & Liquid chip \\
\hline$(38)$ & Interleukin 1 beta $(\mathrm{IL}-1 \beta)$ & $\mathrm{C}$ & Liquid chip \\
\hline$(39)$ & Interleukin 6 (IL-6) & $\mathrm{C}$ & Liquid chip \\
\hline$(40)$ & Interleukin 18 (IL-18) & $\mathrm{C}$ & Liquid chip \\
\hline (41) & Tumor necrosis factor alpha (TNF- $\alpha)$ & $\mathrm{C}$ & Liquid chip \\
\hline$(42)$ & Interleukin 2 (IL-2) & $\mathrm{C}$ & Liquid chip \\
\hline$(43)$ & Interleukin 12 (IL-12) & $\mathrm{C}$ & Liquid chip \\
\hline
\end{tabular}


TABle 1: Continued.

\begin{tabular}{|c|c|c|c|}
\hline Number & Signaling molecule & Classification & Detection method \\
\hline$(44)$ & Interferon gamma (IFN- $\gamma)$ & $\mathrm{C}$ & Liquid chip \\
\hline$(45)$ & Interleukin 4 (IL-4) & $\mathrm{C}$ & Liquid chip \\
\hline$(46)$ & Interleukin 5 (IL-5) & $\mathrm{C}$ & Liquid chip \\
\hline$(47)$ & Interleukin 10 (IL-10) & $\mathrm{C}$ & Liquid chip \\
\hline$(48)$ & Interleukin 17 (IL-17) & $\mathrm{C}$ & Liquid chip \\
\hline$(49)$ & Interleukin 13 (IL-13) & $\mathrm{C}$ & Liquid chip \\
\hline$(50)$ & Interleukin 7 (IL-7) & $\mathrm{C}$ & Liquid chip \\
\hline$(51)$ & Granulocyte-macrophage colony stimulating factor (GM-CSF) & $\mathrm{C}$ & Liquid chip \\
\hline$(52)$ & Macrophage colony-stimulating factor (M-CSF) & $\mathrm{C}$ & Liquid chip \\
\hline$(53)$ & Granulocyte-colony stimulating factor (G-CSF) & $\mathrm{C}$ & Liquid chip \\
\hline$(54)$ & Vascular endothelial growth factor (VEGF) & $\mathrm{C}$ & Liquid chip \\
\hline (55) & C-reactive protein (CRP) & $\mathrm{C}$ & Liquid chip \\
\hline
\end{tabular}

$\mathrm{N}$ : neurotransmitter or neuropeptide; E: endocrine hormone; C: cytokine.

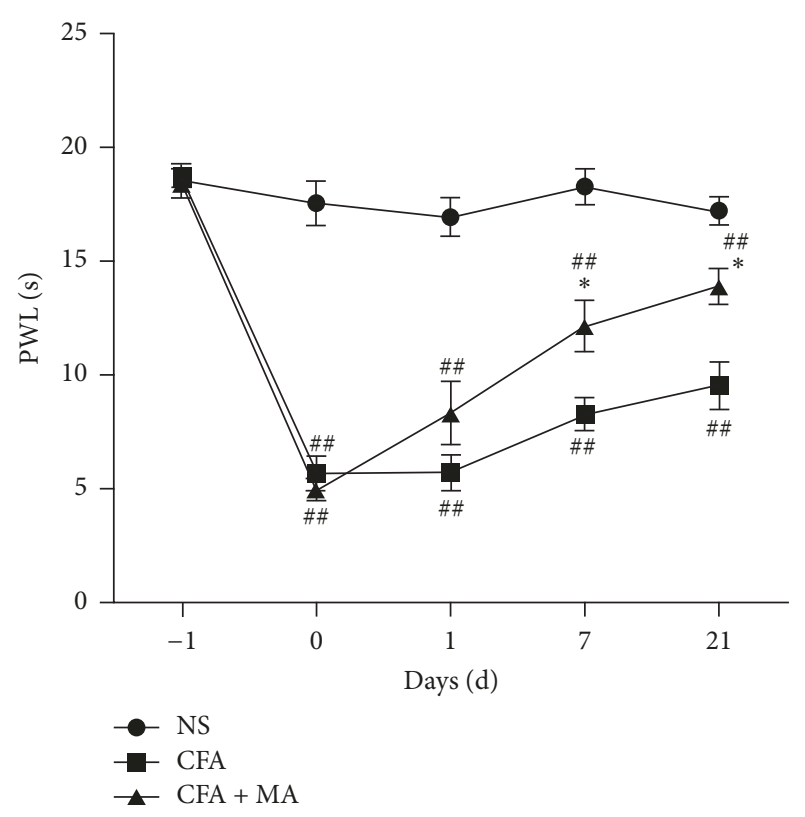

Figure 2: Effect of MA on thermal hyperalgesia. It shows that analgesic effect of MA can be detected on day 7 and day 21 after treatment. $N=7$ per group. ${ }^{\# \#} P<0.01$, CFA versus NS, or CFA + MA versus NS. ${ }^{*} P<0.05, \mathrm{CFA}+\mathrm{MA}$ versus CFA.

groups (NS: $18.62 \pm 2.14 \mathrm{~s}$, CFA: $18.75 \pm 1.26 \mathrm{~s}$, and CFA + MA: $18.47 \pm 1.94 \mathrm{~s})(P>0.05)$. The PWL was significantly lower $(P<0.01)$ in CFA and CFA + MA groups (CFA: $5.70 \pm$ $2.25 \mathrm{~s}, \mathrm{CFA}+\mathrm{MA}: 4.98 \pm 1.47 \mathrm{~s})$ on day 0 after CFA injection than in the NS group $(17.62 \pm 2.76 \mathrm{~s})$. On day 7 and day 21 , in CFA + MA group, PWL was increased significantly after MA $(12.20 \pm 3.37 \mathrm{~s}, 13.95 \pm 2.73 \mathrm{~s}$ on days 7 and 21 , resp.) compared with CFA group $(8.32 \pm 2.18 \mathrm{~s}, 9.60 \pm 3.38 \mathrm{~s}$ on days 7 and 21 , resp. $(P<0.05)$. It indicated that MA had an analgesic effect on inflammatory pain in CFA rats.

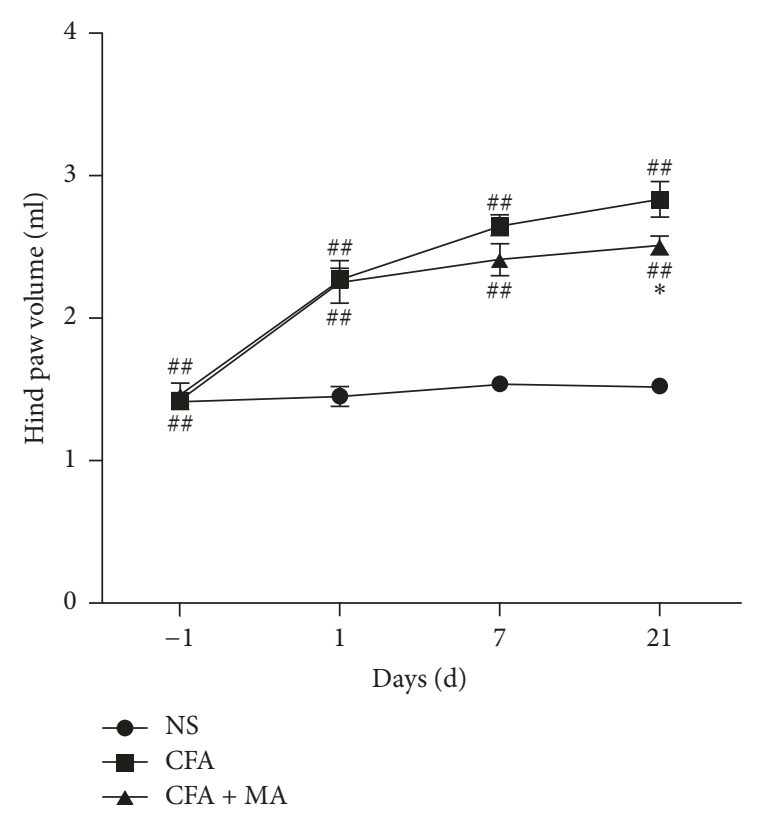

FIGURE 3: Effect of MA on hind paw swelling. A significant antiinflammatory effect of MA on hind paw swelling in CFA rats was observed on day $21 . N=7$ per group. ${ }^{\# \#} P<0.01$, CFA versus NS, or CFA + MA versus NS. ${ }^{*} P<0.05, \mathrm{CFA}+\mathrm{MA}$ versus CFA.

3.2. The Anti-Inflammatory Effect of MA on CFA-Induced Hind Paw Swelling. As shown in Figure 3, there were no differences in hind paw swelling among the three groups prior to CFA injections (NS: $1.42 \pm 0.15 \mathrm{ml}$, CFA: $1.42 \pm 0.14 \mathrm{ml}$, and CFA + MA: $1.47 \pm 0.19 \mathrm{ml})(P>0.05)$. The right hind paw of rats swelled significantly $(P<0.01)$ in CFA and CFA + MA groups (CFA: $2.27 \pm 0.19 \mathrm{ml}, 2.65 \pm 0.18 \mathrm{ml}, 2.84 \pm 0.30 \mathrm{ml}$; CFA + MA: $2.26 \pm 0.36 \mathrm{ml}, 2.42 \pm 0.27 \mathrm{ml}, 2.51 \pm 0.17 \mathrm{ml}$, resp.) on days 1,7 , and 21 after CFA injections compared with the NS group $(1.46 \pm 0.16 \mathrm{ml}, 1.54 \pm 0.13 \mathrm{ml}, 1.53 \pm 0.13 \mathrm{ml}$, 

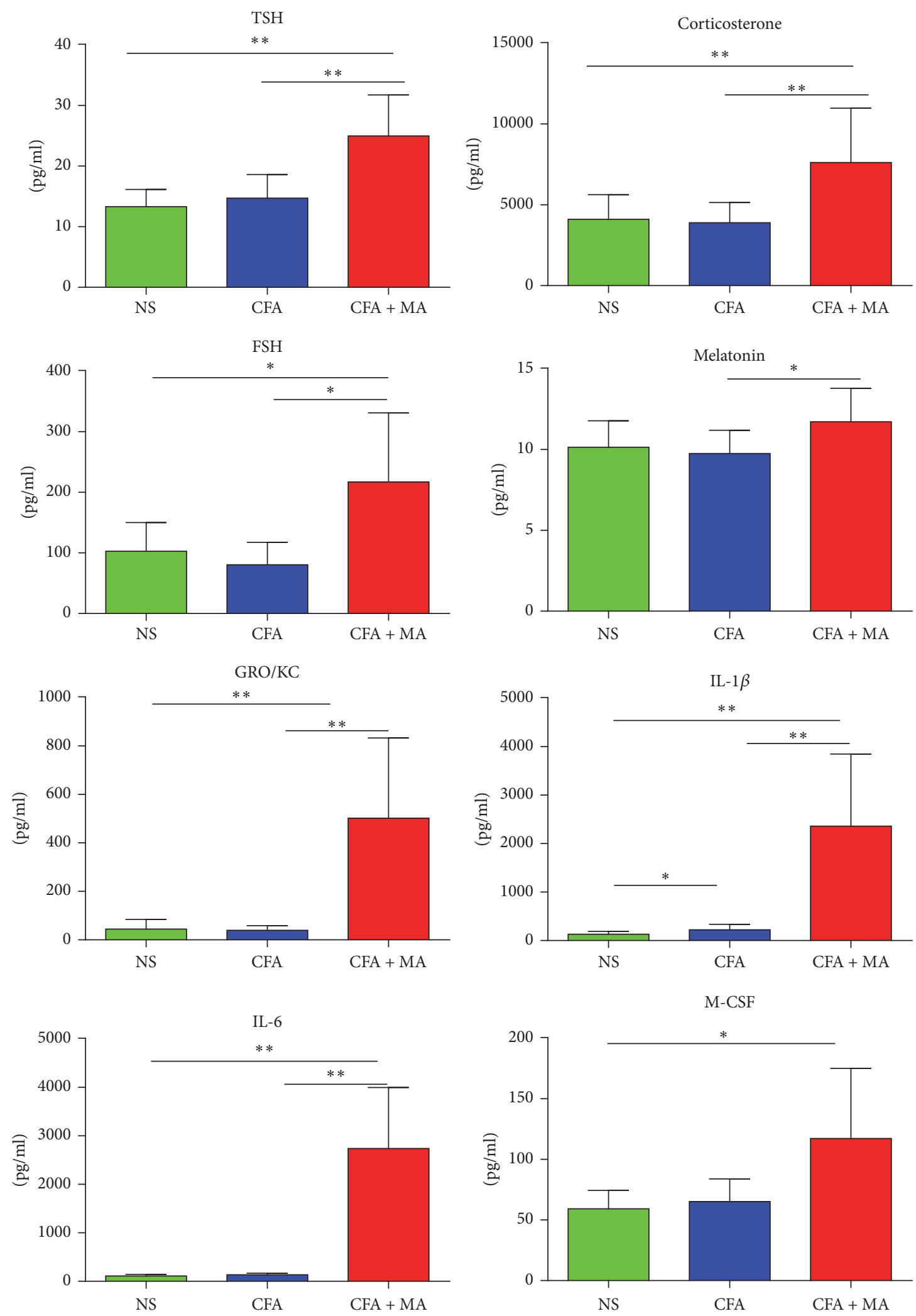

FIGURE 4: Significant changes of the common signaling molecules in the ST36 acupoint induced by MA. A significant statistical differences in common signaling molecules in ST36 acupoint of CFA rats induced by MA was observed on day $21 . N=7$ per group. ${ }^{*} P<0.05$, CFA + MA versus CFA, CFA versus NS, or CFA + MA versus NS. ${ }^{* *} P<0.01, \mathrm{CFA}+\mathrm{MA}$ versus CFA, CFA + MA versus NS. 

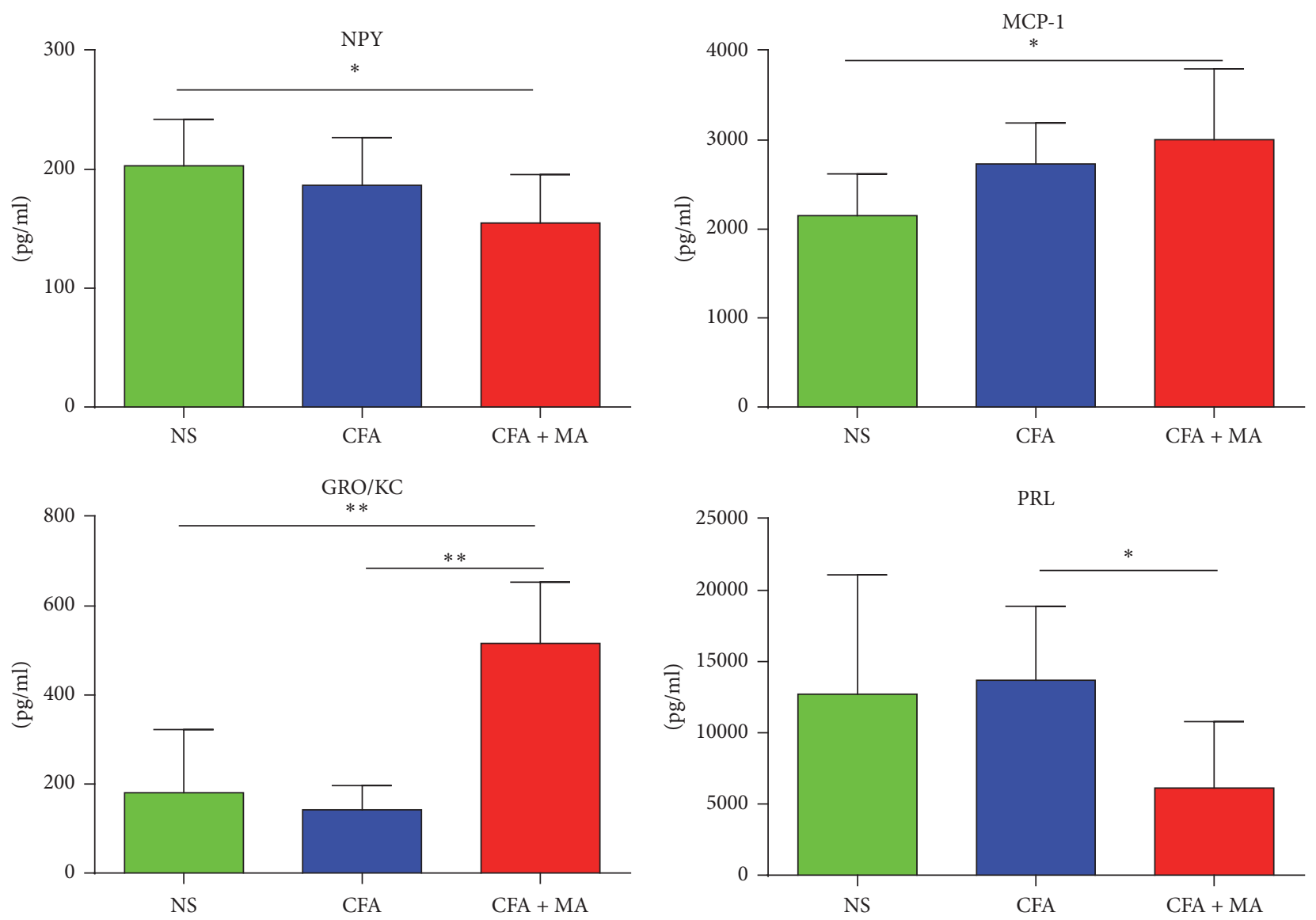

FIGURE 5: Significant changes of common signaling molecules in serum induced by $M A$. A significant statistical differences in common signaling molecules in serum of CFA rats induced by MA was observed on day $21 . N=7$ per group. ${ }^{*} P<0.05$, CFA + MA versus CFA, or CFA + MA versus NS. ${ }^{* *} P<0.01, \mathrm{CFA}+\mathrm{MA}$ versus CFA, or CFA + MA versus NS.

resp.). However, the hind paw swelling significantly decreased $(P<0.05)$ in CFA + MA group $(2.51 \pm 0.17 \mathrm{ml})$ on day 21 compared with CFA group $(2.84 \pm 0.30 \mathrm{ml})$, when MA treatment persisted. These results suggested that MA could alleviate the CFA-induced hind paw swelling.

\subsection{Changes of the Common Signaling Molecules in NEI Network during MA Treatment}

3.3.1. The Changes of Common Signaling Molecules Based on Statistical Analysis. The data with significant statistical differences about the common signaling molecules in the ST36 point, serum, and hind footpad tissue among the three groups on day 21 were separately shown in Figures 4,5 , and 6 . The data without statistical difference among the three groups were not shown in this part. As shown in Figure 4, in the ST36 point, cytokine IL-1 $\beta$ levels were increased in both CFA $(222.79 \pm 111.217 \mathrm{pg} / \mathrm{ml})$ and CFA + MA $(2361.40 \pm 1484.84 \mathrm{pg} / \mathrm{ml})$ groups. In addition, the levels of endocrine hormone TSH, corticosterone, FSH, melatonin $(24.99 \pm 6.78 \mathrm{pg} / \mathrm{ml}, 7620.97 \pm 3364.50 \mathrm{pg} / \mathrm{ml}, 217.25 \pm$ $113.93 \mathrm{pg} / \mathrm{ml}$, and $11.71 \pm 2.08 \mathrm{pg} / \mathrm{ml}$, resp.), and cytokine IL6, GRO/KC $(2738.84 \pm 1260.99 \mathrm{pg} / \mathrm{ml}, 502.40 \pm 330.44 \mathrm{pg} / \mathrm{ml}$, resp.) were upregulated after MA. As shown in Figure 5, in the serum, the level of cytokine $\mathrm{GRO} / \mathrm{KC}$ was upregulated and endocrine hormone PRL was downregulated after MA $(515.95 \pm 137.45 \mathrm{pg} / \mathrm{ml}, 6125.71 \pm 4661.44 \mathrm{pg} / \mathrm{ml}$, resp.). As shown in Figure 6, in the hind footpad tissue, neurotransmitter BDNF and cytokine, namely, RANTES and IL-2 (193.84 \pm $45.32 \mathrm{pg} / \mathrm{ml}, 220.87 \pm 57.96 \mathrm{pg} / \mathrm{ml}$, resp.), level were increased in the CFA group $(43.77 \pm 19.77 \mathrm{pg} / \mathrm{ml})$. In addition, the level of endocrine hormone CRH was upregulated after MA $(0.88 \pm 0.06 \mathrm{pg} / \mathrm{ml})$. These signaling molecules which were identified as statistically significant may be the possible key signaling molecules of NEI network.

3.3.2. The Changes of Common Signaling Molecules Based on Complex Network Analysis. Correlation coefficients between the common signaling molecules of the NEI network in ST36 acupoint, serum, and lateral hind footpad tissue were calculated by Pearson correlation coefficient formula, and signaling moleculars association network (see Figure 7) was constructed based on the signaling molecules chosen by correlation coefficients $\in[-1,-0.8],[0.8,1]$. Then the selected signaling molecules were sorted by node strength correlations, node degree, and node clustering coefficient (see 

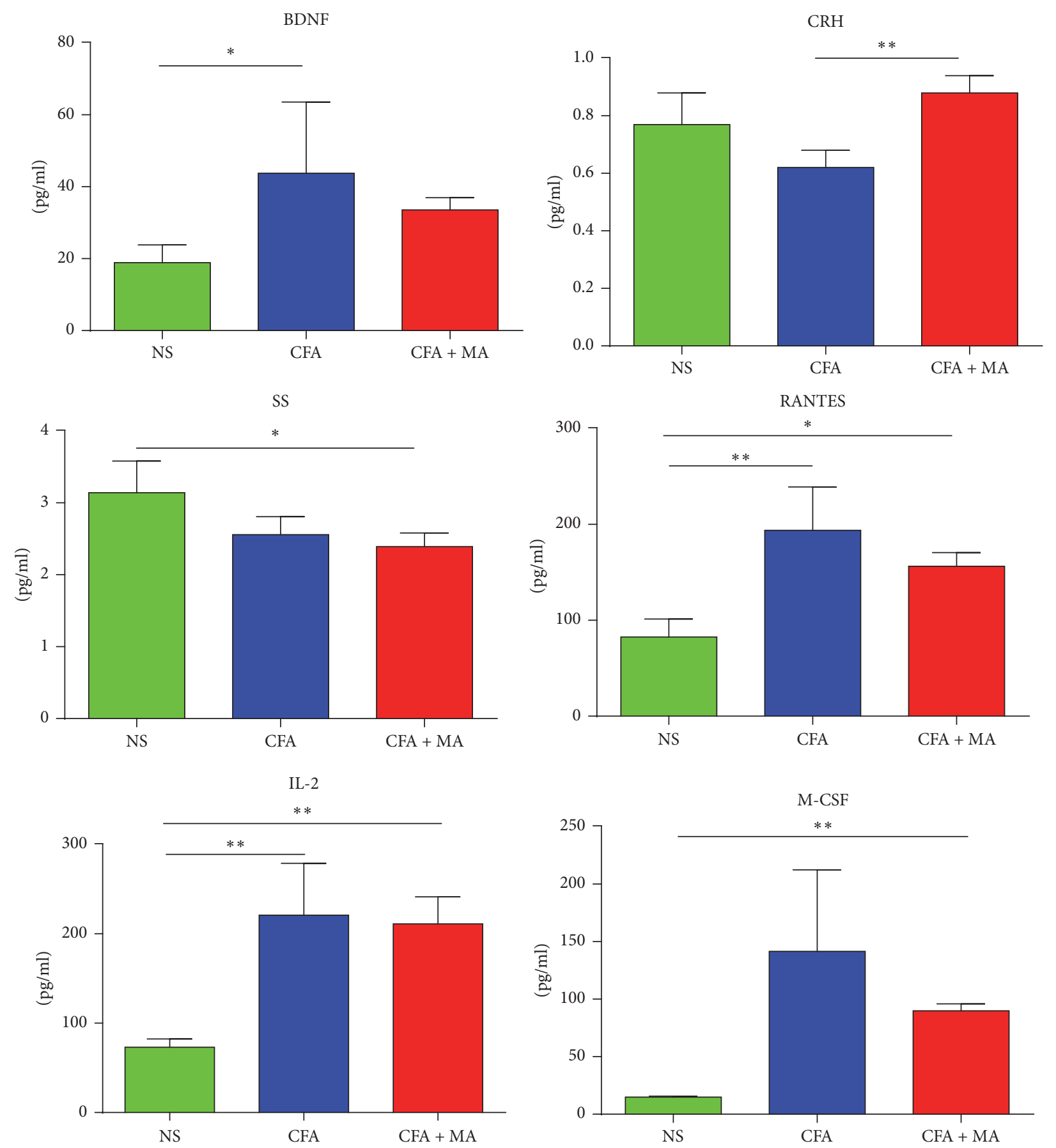

FIGURE 6: Significant changes of common signaling molecules in the hind footpad tissue induced by MA. Significant statistical differences in common signaling molecules in hind footpad tissue of CFA rats induced by MA was observed on day 21 . $N=7$ per group. ${ }^{*} P<0.05$, CFA versus NS, or CFA + MA versus NS. ${ }^{* *} P<0.01$, CFA + MA versus CFA, CFA versus NS, or CFA + MA versus NS.

Tables 2-7.). The first three nodes obtained by analysis with 2 or 3 methods mentioned above were considered as the key signaling molecules in the NEI network (see Table 8).

\section{Discussion}

In this study, the results showed that the PWL obviously decreased and hand paw swelling increased after CFA injections; MA could significantly increase the PWL and decrease hand paw swelling of the CFA rats; it indicated that MA had anti-inflammatory and antinociceptive effect on inflammatory pain in CFA rats. This is consistent with other studies [12-14].

The analysis of statistical results shows that, in CFA group, some common signaling molecules of NEI network in hind foot pad tissue were increased compared with NS 


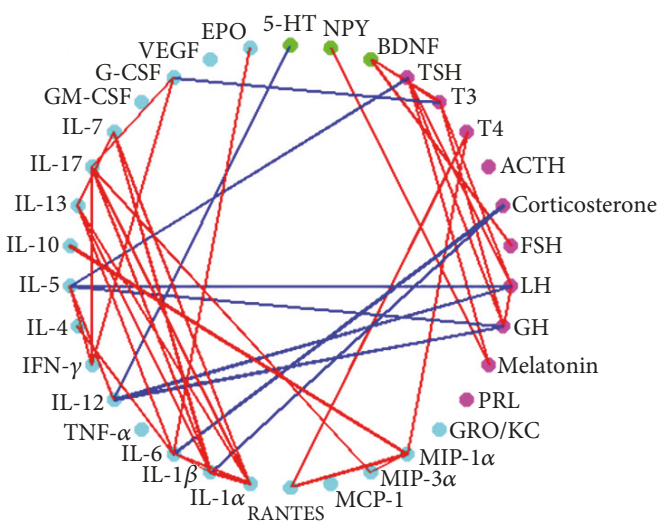

(a)

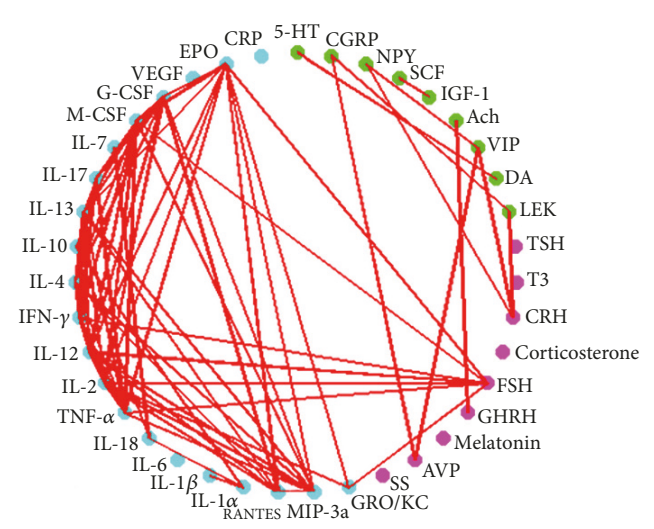

(c)

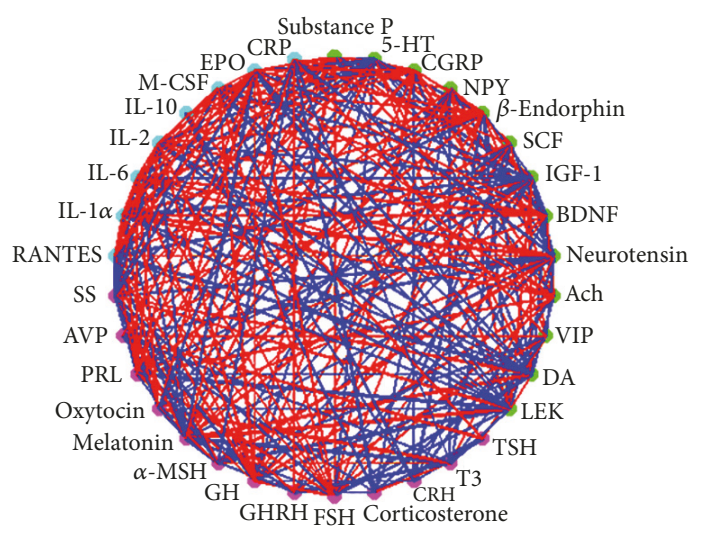

(e)

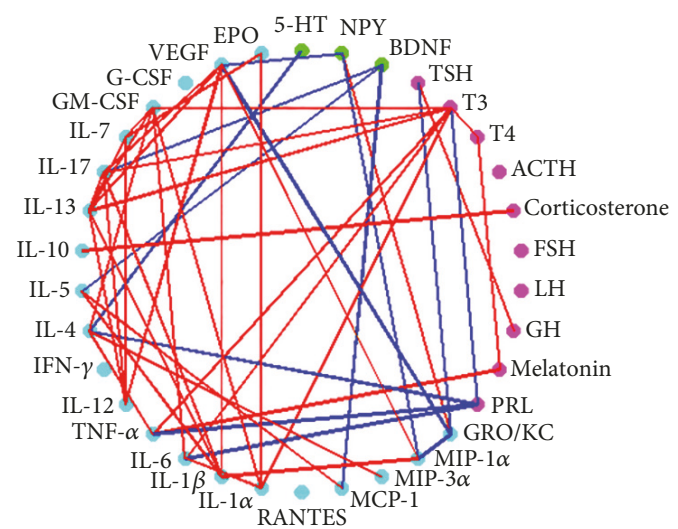

(b)

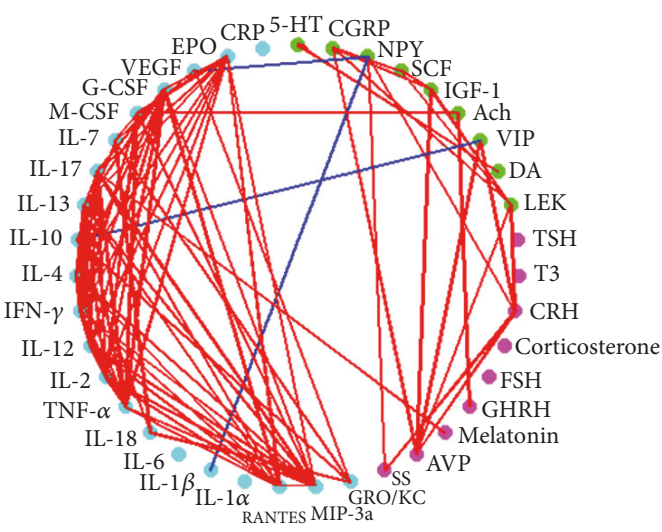

(d)

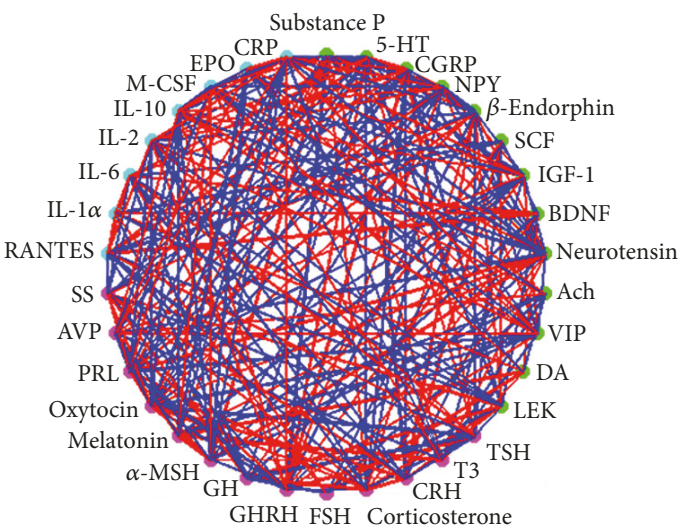

(f)

Figure 7: Common signaling moleculars association network in different samples in groups on day 21 (correlation coefficients $\epsilon$ [-1, -0.8$]$, $[0.8,1])$. Common signaling moleculars association network in serum in CFA group (a) or in CFA + MA group (b). Common signaling moleculars association network in ST36 acupoint in CFA group (c) or in CFA + MA group (d). Common signaling moleculars association network in hind foot pad tissue in CFA group (e) or in CFA + MA group (f). Green dots represent neuropeptide or neurotransmitter, pink dots represent hormone, and blue dots represent cytokines. Red lines represents positive correlation between the molecules; blue lines represent negative correlation between the molecules. The thicker line represents greater correlation coefficient; the thinner line represents smaller correlation coefficient. 
TABLE 2: Common signaling moleculars in serum of CFA group sorted by complex network analysis.

\begin{tabular}{|c|c|c|c|c|c|}
\hline Node strength correlations & Molecular sorting & Node degree & Molecular sorting & Node clustering coefficient & Molecular sorting \\
\hline 5.364006319 & IL-1 $\beta$ & 6 & IL-1 $\beta$ & 0 & $5-\mathrm{HT}$ \\
\hline 5.120790119 & IL-6 & 6 & IL-6 & 0 & NPY \\
\hline 4.464794999 & IL- $1 \alpha$ & 5 & $\mathrm{LH}$ & 0 & $\mathrm{BDNF}$ \\
\hline 4.356832829 & $\mathrm{LH}$ & 5 & $\mathrm{GH}$ & 0 & ACTH \\
\hline 4.356832829 & $\mathrm{GH}$ & 5 & IL- $1 \alpha$ & 0 & FSH \\
\hline 4.177256544 & IL-17 & 5 & IL-5 & 0 & Melatonin \\
\hline 4.09114429 & IL-5 & 5 & IL-17 & 0 & PRL \\
\hline 3.6458827 & IL-13 & 4 & TSH & 0 & $\mathrm{GRO} / \mathrm{KC}$ \\
\hline 3.53653472 & TSH & 4 & $\mathrm{~T} 3$ & 0 & MIP-3 $\alpha$ \\
\hline 3.400877137 & IL-12 & 4 & MIP- $1 \alpha$ & 0 & MCP-1 \\
\hline 3.364263888 & MIP-1 $\alpha$ & 4 & IL-12 & 0 & TNF- $\alpha$ \\
\hline 3.238781977 & $\mathrm{~T} 3$ & 4 & IL-13 & 0 & IL-4 \\
\hline 2.605926586 & G-CSF & 3 & IFN- $\gamma$ & 0 & IL-10 \\
\hline 2.5478666 & IFN- $\gamma$ & 3 & IL-7 & 0 & GM-CSF \\
\hline 2.524251392 & IL-7 & 3 & G-CSF & 0 & VEGF \\
\hline 1.809686502 & Melatonin & 2 & BDNF & 0 & $\mathrm{EPO}$ \\
\hline 1.728692888 & Corticosterone & 2 & $\mathrm{~T} 4$ & 0.166666667 & $\mathrm{~T} 3$ \\
\hline 1.711474914 & MIP-3 $\alpha$ & 2 & Corticosterone & 0.166666667 & MIP-1 $\alpha$ \\
\hline 1.675529628 & RANTES & 2 & Melatonin & 0.2 & IL-17 \\
\hline 1.638782764 & $\mathrm{~T} 4$ & 2 & $\mathrm{MIP}-3 \alpha$ & 0.266666667 & IL-6 \\
\hline 1.60762883 & BDNF & 2 & RANTES & 0.333333333 & IFN- $\gamma$ \\
\hline 0.918065642 & 5-HT & 1 & $5-\mathrm{HT}$ & 0.333333333 & G-CSF \\
\hline 0.865532438 & NPY & 1 & NPY & 0.466666667 & IL-1 $\beta$ \\
\hline 0.837856344 & IL-10 & 1 & FSH & 0.5 & TSH \\
\hline 0.834973817 & IL-4 & 1 & IL-4 & 0.5 & IL-12 \\
\hline 0.816730828 & EPO & 1 & IL-10 & 0.5 & IL-5 \\
\hline 0.806908726 & FSH & 1 & EPO & 0.6 & $\mathrm{LH}$ \\
\hline 0 & ACTH & 0 & $\mathrm{ACTH}$ & 0.6 & $\mathrm{GH}$ \\
\hline 0 & PRL & 0 & PRL & 0.6 & IL- $1 \alpha$ \\
\hline 0 & $\mathrm{GRO} / \mathrm{KC}$ & 0 & $\mathrm{GRO} / \mathrm{KC}$ & 0.833333333 & IL-13 \\
\hline 0 & MCP-1 & 0 & MCP-1 & 1 & $\mathrm{~T} 4$ \\
\hline 0 & TNF- $\alpha$ & 0 & TNF- $\alpha$ & 1 & Corticosterone \\
\hline 0 & GM-CSF & 0 & GM-CSF & 1 & RANTES \\
\hline 0 & VEGF & 0 & VEGF & 1 & IL-7 \\
\hline
\end{tabular}

group, including proinflammatory cytokines RANTES, IL-2, neuropeptide BDNF, indicating that hind footpad tissue after CFA injection was in inflammatory condition. Present studies indicated many similarities regarding the immunological changes and pathologic mechanisms existed between the CFA model and human rheumatoid arthritis (RA). So the CFA model is the widely used animal model for researching mechanisms and therapies of human RA [15]. Cytokines play an important role in pathogenesis of RA, for example, IL-2 could promote inflammatory response, activate macrophages and neutrophils, and inhibit Th2 lymphocyte proliferation in RA [16]. Other studies have found that the chemokine RANTES, secreted by monocytes/macrophages in the synovia of RA patients, was significantly increased [17] and 
TABLE 3: Common signaling moleculars in serum of CFA + MA group sorted by complex network analysis.

\begin{tabular}{|c|c|c|c|c|c|}
\hline Node strength correlations & Molecular sorting & Node degree & Molecular sorting & Node clustering coefficient & Molecular sorting \\
\hline 6.793769656 & $\mathrm{~T} 3$ & 8 & $\mathrm{~T} 3$ & 0 & $5-\mathrm{HT}$ \\
\hline 6.1279229 & IL-13 & 7 & IL-13 & 0 & TSH \\
\hline 6.125755358 & VEGF & 7 & VEGF & 0 & $\mathrm{~T} 4$ \\
\hline 5.345425536 & IL-17 & 6 & IL-17 & 0 & ACTH \\
\hline 5.058280921 & GM-CSF & 6 & GM-CSF & 0 & Corticosterone \\
\hline 4.32642674 & IL- $1 \beta$ & 5 & IL- $1 \beta$ & 0 & FSH \\
\hline 4.23929481 & IL-4 & 5 & IL-4 & 0 & $\mathrm{LH}$ \\
\hline 3.653747121 & MIP-1 $\alpha$ & 4 & PRL & 0 & GH \\
\hline 3.536793987 & $\mathrm{GRO} / \mathrm{KC}$ & 4 & GRO/KC & 0 & Melatonin \\
\hline 3.520423163 & IL-12 & 4 & MIP-1 $1 \alpha$ & 0 & MIP-3 $\alpha$ \\
\hline 3.472198204 & IL-7 & 4 & IL- $1 \alpha$ & 0 & RANTES \\
\hline 3.442092944 & PRL & 4 & IL-6 & 0 & IFN- $\gamma$ \\
\hline 3.437908006 & IL- $1 \alpha$ & 4 & TNF- $\alpha$ & 0 & IL-10 \\
\hline 3.343295878 & IL-6 & 4 & IL-12 & 0 & G-CSF \\
\hline 3.326627841 & TNF- $\alpha$ & 4 & IL-7 & 0 & EPO \\
\hline 2.790223761 & NPY & 3 & NPY & 0.1 & IL-4 \\
\hline 2.628870992 & IL-5 & 3 & $\mathrm{BDNF}$ & 0.25 & $\mathrm{~T} 3$ \\
\hline 2.541007235 & BDNF & 3 & IL-5 & 0.333333333 & BDNF \\
\hline 1.831565026 & $\mathrm{~T} 4$ & 2 & TSH & 0.333333333 & TNF- $\alpha$ \\
\hline 1.810788756 & MCP-1 & 2 & $\mathrm{~T} 4$ & 0.333333333 & IL-5 \\
\hline 1.743848565 & Melatonin & 2 & Melatonin & 0.333333333 & IL-7 \\
\hline 1.73742207 & EPO & 2 & MCP-1 & 0.333333333 & GM-CSF \\
\hline 1.668735302 & TSH & 2 & EPO & 0.4 & IL-1 $\beta$ \\
\hline 0.860173882 & Corticosterone & 1 & 5-HT & 0.4 & IL-17 \\
\hline 0.860173882 & IL-10 & 1 & Corticosterone & 0.428571429 & IL-13 \\
\hline 0.831360077 & $\mathrm{GH}$ & 1 & GH & 0.428571429 & VEGF \\
\hline 0.825950091 & MIP- $3 \alpha$ & 1 & MIP-3 $\alpha$ & 0.5 & PRL \\
\hline 0.811762933 & $5-\mathrm{HT}$ & 1 & IL-10 & 0.5 & $\mathrm{GRO} / \mathrm{KC}$ \\
\hline 0 & ACTH & 0 & ACTH & 0.5 & IL- $1 \alpha$ \\
\hline 0 & FSH & 0 & FSH & 0.6666666667 & MIP-1 $1 \alpha$ \\
\hline 0 & LH & 0 & LH & 0.6666666667 & IL-6 \\
\hline 0 & RANTES & 0 & RANTES & 0.6666666667 & IL-12 \\
\hline 0 & IFN- $\gamma$ & 0 & IFN- $\gamma$ & 1 & NPY \\
\hline 0 & G-CSF & 0 & G-CSF & 1 & MCP-1 \\
\hline
\end{tabular}

could promote osteoclast formation [18], leading to increased inflammation. These were consistent with our results. The increased CRH in hind footpad tissue induced by MA should be further investigated.

Results about the common signaling molecules of NEI network in serum in CFA rats showed that GRO/KC increased and PRL decreased after MA treatment; that still needs to be further studied. Results about the common signaling molecules of NEI network in ST36 acupoint in CFA rats showed that MA induced the increasing expression of some hormones (TSH, melatonin, corticosterone, and FSH), proinflammatory cytokines (IL-1 $\beta$, IL-6), and chemokine $\mathrm{GRO} / \mathrm{KC}$. As MA is a kind of noxious stimulation, so these changes of signaling molecules in ST36 acupoint maybe 
TABLE 4: Common signaling moleculars in ST36 acupoint of CFA group sorted by complex network analysis.

\begin{tabular}{|c|c|c|c|c|c|}
\hline Node strength correlations & Molecular sorting & Node degree & Molecular sorting & Node clustering coefficient & Molecular sorting \\
\hline 10.72648569 & IL-2 & 12 & IL-2 & 0 & 5-HT \\
\hline 9.92879842 & IL-12 & 11 & IL-12 & 0 & CGRP \\
\hline 9.756829776 & IL-13 & 11 & IL-13 & 0 & SCF \\
\hline 9.692100786 & M-CSF & 11 & M-CSF & 0 & IGF-1 \\
\hline 8.865265516 & EPO & 10 & TNF- $\alpha$ & 0 & Ach \\
\hline 8.864817973 & IL-4 & 10 & IL-4 & 0 & DA \\
\hline 8.756035321 & TNF- $\alpha$ & 10 & EPO & 0 & LEK \\
\hline 8.190175267 & MIP-3 $\alpha$ & 9 & MIP-3 $\alpha$ & 0 & TSH \\
\hline 7.868172048 & G-CSF & 9 & G-CSF & 0 & $\mathrm{~T} 3$ \\
\hline 6.823300188 & IFN- $\gamma$ & 8 & IFN- $\gamma$ & 0 & Corticosterone \\
\hline 6.281832099 & RANTES & 7 & FSH & 0 & GHRH \\
\hline 6.133378999 & FSH & 7 & RANTES & 0 & Melatonin \\
\hline 5.071780915 & IL-17 & 6 & IL-17 & 0 & AVP \\
\hline 2.725513459 & IL-18 & 3 & VIP & 0 & SS \\
\hline 2.697281121 & $\mathrm{CRH}$ & 3 & $\mathrm{CRH}$ & 0 & IL-1 $\beta$ \\
\hline 2.695360587 & VIP & 3 & GRO/KC & 0 & IL-6 \\
\hline 2.670612454 & GRO/KC & 3 & IL- $1 \alpha$ & 0 & VEGF \\
\hline 2.614138569 & IL-10 & 3 & IL-18 & 0 & CRP \\
\hline 2.549577048 & IL- $1 \alpha$ & 3 & IL-10 & 0.333333333 & VIP \\
\hline 1.798304838 & LEK & 2 & CGRP & 0.333333333 & $\mathrm{CRH}$ \\
\hline 1.758387897 & CGRP & 2 & NPY & 0.333333333 & IL- $1 \alpha$ \\
\hline 1.757512896 & NPY & 2 & LEK & 0.666666667 & IL-18 \\
\hline 1.711380393 & AVP & 2 & AVP & 0.666666667 & IL-2 \\
\hline 1.704535511 & IL-7 & 2 & IL-7 & 0.666666667 & IL-10 \\
\hline 0.999281664 & $5-\mathrm{HT}$ & 1 & $5-\mathrm{HT}$ & 0.688888889 & EPO \\
\hline 0.999281664 & DA & 1 & SCF & 0.745454545 & M-CSF \\
\hline 0.870807119 & IL- $1 \beta$ & 1 & IGF-1 & 0.755555556 & TNF- $\alpha$ \\
\hline 0.863267828 & Ach & 1 & Ach & 0.761904762 & FSH \\
\hline 0.863267828 & GHRH & 1 & DA & 0.763636364 & IL-12 \\
\hline 0.832906845 & SCF & 1 & GHRH & 0.763636364 & IL-13 \\
\hline 0.832906845 & IGF-1 & 1 & IL- $1 \beta$ & 0.777777778 & IL-4 \\
\hline 0 & TSH & 0 & TSH & 0.805555556 & G-CSF \\
\hline 0 & T3 & 0 & $\mathrm{~T} 3$ & 0.821428571 & IFN- $\gamma$ \\
\hline 0 & Corticosterone & 0 & Corticosterone & 0.866666667 & IL-17 \\
\hline 0 & Melatonin & 0 & Melatonin & 0.888888889 & MIP-3 $\alpha$ \\
\hline 0 & SS & 0 & SS & 0.952380952 & RANTES \\
\hline 0 & IL-6 & 0 & IL-6 & 1 & NPY \\
\hline 0 & VEGF & 0 & VEGF & 1 & GRO/KC \\
\hline 0 & CRP & 0 & CRP & 1 & IL-7 \\
\hline
\end{tabular}


TABLE 5: Common signaling moleculars in ST36 acupoint of CFA + MA group sorted by complex network analysis.

\begin{tabular}{|c|c|c|c|c|c|}
\hline Node strength correlations & Molecular sorting & Node degree & Molecular sorting & Node clustering coefficient & Molecular sorting \\
\hline 10.30508457 & IFN- $\gamma$ & 11 & MIP-3 $\alpha$ & 0 & $5-\mathrm{HT}$ \\
\hline 10.20967738 & MIP-3 $\alpha$ & 11 & TNF- $\alpha$ & 0 & Ach \\
\hline 10.14565087 & TNF- $\alpha$ & 11 & IL-2 & 0 & DA \\
\hline 10.14143505 & $\mathrm{EPO}$ & 11 & IFN- $\gamma$ & 0 & TSH \\
\hline 9.984491306 & IL-2 & 11 & IL-17 & 0 & T3 \\
\hline 9.835412633 & IL-17 & 11 & EPO & 0 & Corticosterone \\
\hline 9.586116688 & IL-4 & 10 & RANTES & 0 & FSH \\
\hline 9.58555599 & IL-13 & 10 & IL-12 & 0 & GHRH \\
\hline 9.496416209 & IL-12 & 10 & IL-4 & 0 & Melatonin \\
\hline 9.324953981 & G-CSF & 10 & IL-13 & 0 & IL- $1 \alpha$ \\
\hline 9.322535406 & RANTES & 10 & G-CSF & 0 & IL-1 $\beta$ \\
\hline 5.816288172 & M-CSF & 7 & M-CSF & 0 & IL-6 \\
\hline 4.447630823 & IL-7 & 5 & LEK & 0 & CRP \\
\hline 4.428353692 & LEK & 5 & $\mathrm{CRH}$ & 0.166666667 & NPY \\
\hline 4.330583532 & AVP & 5 & AVP & 0.3 & $\mathrm{CRH}$ \\
\hline 4.254168273 & $\mathrm{CRH}$ & 5 & IL-7 & 0.333333333 & VIP \\
\hline 3.726312879 & CGRP & 4 & CGRP & 0.333333333 & VEGF \\
\hline 3.665515648 & $\mathrm{GRO} / \mathrm{KC}$ & 4 & NPY & 0.4 & IL-7 \\
\hline 3.631586867 & IGF-1 & 4 & IGF-1 & 0.476190476 & M-CSF \\
\hline 3.626838652 & IL-10 & 4 & $\mathrm{GRO} / \mathrm{KC}$ & 0.5 & AVP \\
\hline 3.3417145 & NPY & 4 & IL-10 & 0.5 & IL-10 \\
\hline 2.863080058 & IL-18 & 3 & SCF & 0.6 & LEK \\
\hline 2.733155888 & SCF & 3 & VIP & 0.666666667 & $\mathrm{GRO} / \mathrm{KC}$ \\
\hline 2.612860185 & VIP & 3 & IL-18 & 0.818181818 & IL-17 \\
\hline 2.432869853 & VEGF & 3 & VEGF & 0.833333333 & CGRP \\
\hline 1.777426407 & Ach & 2 & Ach & 0.833333333 & IGF-1 \\
\hline 1.745137778 & SS & 2 & SS & 0.890909091 & MIP-3 $\alpha$ \\
\hline 0.995761145 & $5-\mathrm{HT}$ & 1 & $5-\mathrm{HT}$ & 0.890909091 & TNF- $\alpha$ \\
\hline 0.995761145 & DA & 1 & DA & 0.890909091 & IL-2 \\
\hline 0.909867657 & GHRH & 1 & GHRH & 0.890909091 & IFN- $\gamma$ \\
\hline 0.832121031 & IL-1 $\beta$ & 1 & Melatonin & 0.890909091 & EPO \\
\hline 0.831984815 & Melatonin & 1 & IL-1 $\beta$ & 1 & SCF \\
\hline 0 & $\mathrm{TSH}$ & 0 & TSH & 1 & SS \\
\hline 0 & T3 & 0 & $\mathrm{~T} 3$ & 1 & RANTES \\
\hline 0 & Corticosterone & 0 & Corticosterone & 1 & IL-18 \\
\hline 0 & FSH & 0 & FSH & 1 & IL-12 \\
\hline 0 & IL- $1 \alpha$ & 0 & IL-1 $\alpha$ & 1 & IL-4 \\
\hline 0 & IL-6 & 0 & IL-6 & 1 & IL-13 \\
\hline 0 & CRP & 0 & CRP & 1 & G-CSF \\
\hline
\end{tabular}


TABLE 6: Common signaling moleculars in hind footpad tissue of CFA group sorted by complex network analysis.

\begin{tabular}{|c|c|c|c|c|c|}
\hline Node strength correlations & Molecular sorting & Node degree & Molecular sorting & Node clustering coefficient & Molecular sorting \\
\hline 19.10661085 & $\mathrm{EPO}$ & 20 & Substance P & 0.5 & TSH \\
\hline 19.10443745 & Substance P & 20 & $5-\mathrm{HT}$ & 0.5 & IL-10 \\
\hline 19.09503987 & IL- $1 \alpha$ & 20 & IGF-1 & 0.588235294 & Neurotensin \\
\hline 19.08824776 & IL-6 & 20 & LEK & 0.607142857 & $\mathrm{CRH}$ \\
\hline 19.07123329 & IGF-1 & 20 & Melatonin & 0.617647059 & Oxytocin \\
\hline 19.06447235 & $5-\mathrm{HT}$ & 20 & IL- $1 \alpha$ & 0.631578947 & Melatonin \\
\hline 18.50160671 & LEK & 20 & IL-6 & 0.666666667 & Corticosterone \\
\hline 18.29600965 & PRL & 20 & $\mathrm{EPO}$ & 0.692307692 & $\alpha-\mathrm{MSH}$ \\
\hline 18.29571712 & IL-2 & 19 & $\beta$-Endorphin & 0.705263158 & LEK \\
\hline 18.27023516 & FSH & 19 & DA & 0.772727273 & NPY \\
\hline 18.25430456 & DA & 19 & FSH & 0.772727273 & T3 \\
\hline 18.25266497 & Melatonin & 19 & PRL & 0.782051282 & CGRP \\
\hline 18.0674304 & M-CSF & 19 & SS & 0.8 & VIP \\
\hline 18.04781731 & $\beta$-Endorphin & 19 & RANTES & 0.8 & AVP \\
\hline 17.94864817 & SS & 19 & IL-2 & 0.807017544 & SS \\
\hline 17.94592524 & RANTES & 19 & M-CSF & 0.807017544 & RANTES \\
\hline 16.95153766 & $\mathrm{GH}$ & 18 & $\mathrm{GH}$ & 0.831578947 & $5-\mathrm{HT}$ \\
\hline 15.47470248 & Oxytocin & 17 & Neurotensin & 0.831578947 & IGF-1 \\
\hline 15.40534815 & Neurotensin & 17 & Oxytocin & 0.831578947 & IL- $1 \alpha$ \\
\hline 14.93565299 & Ach & 16 & BDNF & 0.831578947 & IL-6 \\
\hline 14.9351783 & BDNF & 16 & Ach & 0.836363636 & SCF \\
\hline 12.16893349 & $\alpha-\mathrm{MSH}$ & 13 & CGRP & 0.836363636 & CRP \\
\hline 11.68002365 & CGRP & 13 & $\alpha-\mathrm{MSH}$ & 0.842105263 & Substance P \\
\hline 11.2866825 & NPY & 12 & NPY & 0.842105263 & $\beta$-Endorphin \\
\hline 11.27743293 & T3 & 12 & T3 & 0.842105263 & M-CSF \\
\hline 10.44668172 & CRP & 11 & SCF & 0.842105263 & EPO \\
\hline 10.43829458 & SCF & 11 & CRP & 0.85620915 & $\mathrm{GH}$ \\
\hline 9.545156525 & GHRH & 10 & VIP & 0.883040936 & DA \\
\hline 9.391637867 & AVP & 10 & GHRH & 0.883040936 & FSH \\
\hline 9.373485793 & VIP & 10 & AVP & 0.883040936 & PRL \\
\hline 8.148967846 & Corticosterone & 9 & Corticosterone & 0.883040936 & IL-2 \\
\hline 7.229380481 & $\mathrm{CRH}$ & 8 & $\mathrm{CRH}$ & 0.883333333 & $\mathrm{BDNF}$ \\
\hline 4.484931739 & TSH & 5 & TSH & 0.883333333 & Ach \\
\hline 3.693908813 & IL-10 & 4 & IL-10 & 0.888888889 & GHRH \\
\hline
\end{tabular}

the normal responses to noxious stimulation and specific responses to MA. Zhang et al. proposed the concept of Neural Acupuncture Unit: acupuncture could excite nerve in the local acupoint and could also activate the cells closely connected with the nerve, so as to promote the release of neurotransmitters, hormones, and cytokines, and then transfer the acupuncture information [19]. Hi-Joon Par found that some neural and immune pathways, such as MAPK, B-cell receptor, T-cell receptor, and Toll-like receptor, in the local acupoint were involved in the anti-inflammatory and antinociceptive effect of acupuncture on inflammatory pain in CFA rats [20]. These findings support our results. 
TABLE 7: Common signaling moleculars in hind foot pad tissue of CFA + MA group sorted by complex network analysis.

\begin{tabular}{|c|c|c|c|c|c|}
\hline Node strength correlations & Molecular sorting & Node degree & Molecular sorting & Node clustering coefficient & Molecular sorting \\
\hline 18.40435568 & Substance P & 20 & Substance P & 0.5 & DA \\
\hline 18.40150748 & Neurotensin & 20 & Neurotensin & 0.642857143 & FSH \\
\hline 17.63806473 & Oxytocin & 19 & Oxytocin & 0.666666667 & LEK \\
\hline 17.60762122 & PRL & 19 & PRL & 0.69005848 & Oxytocin \\
\hline 16.88856039 & GHRH & 18 & NPY & 0.692810458 & $\beta$-Endorphin \\
\hline 16.83720135 & NPY & 18 & $\beta$-Endorphin & 0.7 & Substance P \\
\hline 16.79938918 & CRP & 18 & GHRH & 0.7 & Neurotensin \\
\hline 16.69891691 & $\beta$-Endorphin & 18 & CRP & 0.705128205 & IL-6 \\
\hline 16.00352427 & VIP & 17 & $5-\mathrm{HT}$ & 0.705882353 & $5-\mathrm{HT}$ \\
\hline 15.77571079 & IL-2 & 17 & CGRP & 0.705882353 & Corticosterone \\
\hline 15.69108923 & CGRP & 17 & VIP & 0.713235294 & CGRP \\
\hline 15.65507789 & Corticosterone & 17 & Corticosterone & 0.713450292 & PRL \\
\hline 15.6462691 & $5-\mathrm{HT}$ & 17 & IL-2 & 0.714285714 & RANTES \\
\hline 15.20421904 & IGF-1 & 16 & IGF-1 & 0.720588235 & IL-2 \\
\hline 15.157233 & $\alpha-\mathrm{MSH}$ & 16 & $\alpha-\mathrm{MSH}$ & 0.722222222 & Ach \\
\hline 15.15132027 & AVP & 16 & AVP & 0.725490196 & GHRH \\
\hline 15.04667388 & IL-10 & 16 & IL-10 & 0.727272727 & $\mathrm{GH}$ \\
\hline 13.98760052 & SS & 15 & LEK & 0.736263736 & $\mathrm{CRH}$ \\
\hline 13.80904047 & LEK & 15 & SS & 0.738562092 & NPY \\
\hline 13.77891314 & M-CSF & 15 & M-CSF & 0.75 & $\mathrm{BDNF}$ \\
\hline 13.1712101 & $\mathrm{TSH}$ & 14 & TSH & 0.752380952 & SS \\
\hline 13.1430236 & $\mathrm{CRH}$ & 14 & $\mathrm{CRH}$ & 0.758169935 & CRP \\
\hline 13.09659812 & RANTES & 14 & Melatonin & 0.758241758 & TSH \\
\hline 12.88753949 & Melatonin & 14 & RANTES & 0.785714286 & IL- $1 \alpha$ \\
\hline 11.9691417 & IL-6 & 13 & $\mathrm{~T} 3$ & 0.79047619 & $\mathrm{M}-\mathrm{CSF}$ \\
\hline 11.905127 & $\mathrm{~T} 3$ & 13 & IL-6 & 0.794117647 & VIP \\
\hline 11.89354354 & EPO & 13 & EPO & 0.813186813 & Melatonin \\
\hline 11.08226901 & $\mathrm{GH}$ & 12 & $\mathrm{GH}$ & 0.816666667 & $\alpha-\mathrm{MSH}$ \\
\hline 8.51846022 & SCF & 9 & SCF & 0.816666667 & AVP \\
\hline 8.373711747 & $\mathrm{BDNF}$ & 9 & BDNF & 0.816666667 & IL-10 \\
\hline 8.142776395 & Ach & 9 & Ach & 0.833333333 & SCF \\
\hline 7.508261048 & IL- $1 \alpha$ & 8 & DA & 0.833333333 & IGF-1 \\
\hline 7.278849151 & FSH & 8 & FSH & 0.833333333 & $\mathrm{~T} 3$ \\
\hline 7.056443065 & DA & 8 & IL- $1 \alpha$ & 0.833333333 & EPO \\
\hline
\end{tabular}

The changes of these molecules may play a key role in the production and transmission of acupuncture information [21]. It still needs further research in the future.

The analysis of complex network results shows that, in serum, IL- $1 \beta$, IL- 6 , and IL- $1 \alpha$ were the key signaling molecules in CFA rats, and T3, IL-13, and VEGF were the key signaling molecules in CFA + MA rats. Moreover, the key signaling molecules in hind footpad tissue of CFA rats were EPO, SP, and IL- $1 \alpha$ and in CFA + MA rats were substance $\mathrm{P}$, neurotensin, and oxytocin. These indicate that the key signaling molecules we acquired were consistent with the pathogenesis of RA and the analgesic effect and antiinflammatory mechanism of MA. Some researches reported that IL-2, IL-12, IL- $1 \alpha$, IL-1 $\beta$, IL- 6 , and TNF- $\alpha$ could induce 
TABLE 8: Key common signaling moleculars in different samples based on complex network analysis.

\begin{tabular}{lccc}
\hline & Serum & ST36 acupoint & Hind footpad tissue \\
\hline \multirow{4}{*}{ CFA } & IL-1 $\beta$ & IL-2 & EPO \\
& IL-6 & IL-12 & Substance P \\
& IL-1 $\alpha$ & IL-13 & IL-1 $\alpha$ \\
\hline \multirow{4}{*}{ CFA + MA } & T3 & IFN- $\gamma$ & Substance P \\
& IL-13 & MIP-3 $\alpha$ & Neurotensin \\
& VEGF & TNF- $\alpha$ & Oxytocin \\
\hline
\end{tabular}

acute phase reaction, stimulate the growth and differentiation of hematopoietic precursor cells, promote the proliferation of synovial fibroblasts, and cause joint damage [22] in the pathogenesis of RA. IL-13, produced by activated Th2 cells, had anti-inflammatory and immunomodulatory effects [23]. Therefore, these signaling molecules may play a key role in acupuncture regulating the NEI network but require further experimental confirmation. Other key signaling molecules identified, such as VEGF, EPO, SP, NT, and OT, need further experiments to explain the result. In the ST36 acupoint, the results of complex network showed that the key signaling molecules after MA were IFN- $\gamma$, MIP- $3 \alpha$, and TNF- $\alpha$, and the key signaling molecules identified by statistical methods included IL- $1 \beta$, IL-6, and GRO/KC, which belong to proinflammatory cytokines or chemokines, so we hypothesize that acupuncture, as a physical stimulus, may cause an inflammatory reaction in the local acupoint, amplify the acupuncture information in cascade, and act on the NEI network, eventually producing acupuncture effects. The inflammatory response induced by acupuncture may be the starting point of acupuncture effect. In the future we will further research this part.

In this study, we detected 55 kinds of common signaling molecules of NEI network in three parts of CFA rats, including serum, ST36 acupoint, and hind footpad tissue. Since some signaling molecules in the three parts failed to be detected by the measurement techniques, the types and numbers of the signaling molecules detected in three parts were different. So it may affect the analysis results.

\section{Conclusions}

In conclusion, this study shows that MA has obvious analgesic and anti-inflammatory effects on CFA rats with inflammatory pain; the key signaling molecules of ST36 acupoint, serum, and hind footpad tissue acquired by statistical and complex network methods were all consistent with the pathogenesis of RA and the analgesic effect and anti-inflammatory mechanism of MA; these key signaling molecules in the three parts may play an important role in MA modulating NEI network; it still needs to be further studied in the future.

\section{Conflicts of Interest}

The authors declare that they have no conflicts of interest.

\section{Authors' Contributions}

Yi Guo, Jiang Wang, and Yang-yang Liu conceived and designed the study. Sha-sha Ding, Ya-wen Yan, and Kuo Zhang performed the experiments. Kuo Zhang and Xinmeng Guo wrote the paper. Zhi-fang Xu, Xue Zhao, and Kai Li reviewed and edited the manuscript. All authors read and approved the manuscript. Kuo Zhang and Xin-meng Guo contributed equally to this work.

\section{Acknowledgments}

This work was supported by the National Natural Science Foundation of China (NSFC), Grants nos. 81330088 and 81603677.

\section{References}

[1] J. E. Blalock, "The syntax of immune-neuroendocrine communication," Trends in Immunology, vol. 15, no. 11, pp. 504-511, 1994.

[2] E. M. Smith and J. E. Blalock, "A molecular basis for interactions between the immune and neuroendocrine systems," International Journal of Neuroscience, vol. 38, no. 3-4, pp. 455-464, 1988.

[3] S. S. Ding, S. H. Hong, C. Wang, Y. Guo, Z. K. Wang, and $\mathrm{Y}$. Xu, "Acupuncture modulates the neuro-endocrine-immune network," QJM: An International Journal of Medicine, vol. 107, no. 5, pp. 341-345, 2014.

[4] H. Besedovsky and E. Sorkin, "Network of immune-neuroendocrine interactions," Clinical \& Experimental Immunology, vol. 27, pp. 1-12, 1977.

[5] S.-Y. Cui and C.-Z. Tang, "Progress in the research of neuroendocrine-immunological mechanism of acupuncture in regulating blood pressure and protecting target organs in hypertension," Acupuncture Research, vol. 33, no. 3, pp. 208-212, 2008.

[6] B. Chen, M. Li, S. Dind et al., "Research progress on regulations on nerve-endocrine-immune network by acupuncture," World Journal of Acupuncture - Moxibustion, vol. 24, no. 4, pp. 49-58, 2014.

[7] W. L. Lanier, "Mayo clinic proceedings 2013: Report from a new vista," Mayo Clinic Proceedings, vol. 88, no. 1, pp. 7-10, 2013.

[8] D. J. Watts and S. H. Strogatz, "Collective dynamics of 'smallworld' networks," Nature, vol. 393, no. 6684, pp. 440-442, 1998.

[9] A.-L. Barabasi and R. Albert, "Emergence of scaling in random networks," American Association for the Advancement of Science: Science, vol. 286, no. 5439, pp. 509-512, 1999.

[10] Y. Wang, R. Gehringer, S. A. Mousa, D. Hackel, A. Brack, and H. L. Rittner, "CXCL10 controls inflammatory pain via opioid peptide-containing macrophages in electroacupuncture," PLoS ONE, vol. 9, no. 4, Article ID e94696, 2014.

[11] N. Shejawal, S. Menon, and S. Shailajan, "A simple, sensitive and accurate method for rat paw volume measurement and its expediency in preclinical animal studies," Human \& Experimental Toxicology, vol. 33, no. 2, pp. 123-129, 2014.

[12] W.-L. Mi, Q.-L. Mao-Ying, X.-W. Wang et al., "Involvement of spinal neurotrophin-3 in electroacupuncture analgesia and inhibition of spinal glial activation in rat model of monoarthritis," The Journal of Pain, vol. 12, no. 9, pp. 974-984, 2011. 
[13] T.-F. He, S.-H. Zhang, L.-B. Li, W.-J. Yang, J. Zhu, and Y.-F. Chen, "Effects of acupuncture on the number and degranulation ratio of mast cells and expression of tryptase in synovium of rats with adjuvant arthritis," Journal of Chinese Integrative Medicine, vol. 8, no. 7, pp. 670-677, 2010.

[14] H. Huang, R. Zhan, X.-J. Yu, D. Zhang, W.-M. Li, and G.-H. Ding, "Effects of acupoint-nerve block on mast cell activity, manual acupuncture- and electroacupuncture-induced analgesia in adjuvant arthritis rats," Acupuncture Research, vol. 34, no. 1, pp. 31-56, 2009.

[15] P. Gohil, V. Patel, S. Deshpande, M. Chorawala, and G. Shah, "Anti-arthritic activity of cell wall content of Lactobacillus plantarum in freund's adjuvant-induced arthritic rats: involvement of cellular inflammatory mediators and other biomarkers," Inflammopharmacology, pp. 1-11, 2017.

[16] E. Brzustewicz and E. Bryl, "The role of cytokines in the pathogenesis of rheumatoid arthritis - Practical and potential application of cytokines as biomarkers and targets of personalized therapy," Cytokine, vol. 76, no. 2, pp. 527-536, 2015.

[17] S. A. Agere, N. Akhtar, J. M. Watson, and S. Ahmed, "RANTES/CCL5 Induces Collagen Degradation by Activating MMP-1 and MMP-13 Expression in Human Rheumatoid Arthritis Synovial Fibroblasts," Frontiers in Immunology, vol. 8, article 1341, 2017.

[18] A. Sucur, Z. Jajic, M. Artukovic et al., "Chemokine signals are crucial for enhanced homing and differentiation of circulating osteoclast progenitor cells," Arthritis Research \& Therapy, vol. 19, no. 1, article no. 142, 2017.

[19] Z. J. Zhang, X. M. Wang, and G. M. McAlonan, "Neural acupuncture unit: a new concept for interpreting effects and mechanisms of acupuncture," Evidence-Based Complementary and Alternative Medicine, vol. 2012, Article ID 429412, 23 pages, 2012.

[20] J.-Y. Park, J. J. Park, S. Jeon et al., "From peripheral to central: the role of ERK signaling pathway in acupuncture analgesia," The Journal of Pain, vol. 15, no. 5, pp. 535-549, 2014.

[21] N. Goldman, M. Chen, T. Fujita et al., "Adenosine A1 receptors mediate local anti-nociceptive effects of acupuncture," Nature Neuroscience, vol. 13, no. 7, pp. 883-888, 2010.

[22] E. H. S. Choy and G. S. Panayi, "Cytokine pathways and joint inflamation in rheumatoid arthritis," The New England Journal of Medicine, vol. 344, no. 12, pp. 907-916, 2001.

[23] K. Fukuda, Y. Miura, T. Maeda, M. Takahashi, S. Hayashi, and M. Kurosaka, "Decoy receptor 3 regulates the expression of various genes in rheumatoid arthritis synovial fibroblasts," International Journal of Molecular Medicine, vol. 32, no. 4, pp. 910-916, 2013. 


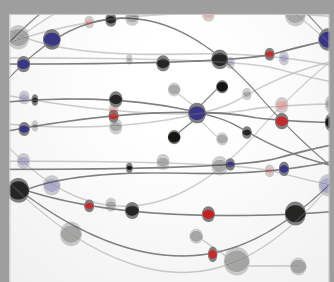

The Scientific World Journal
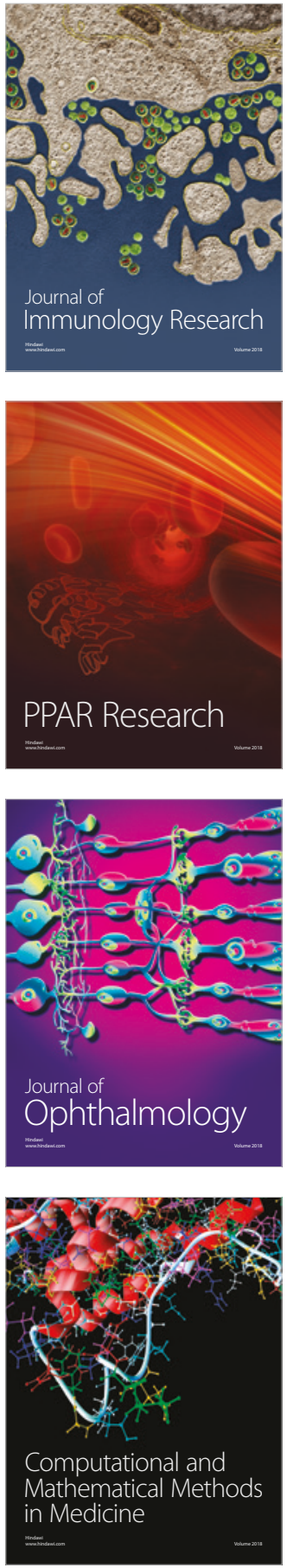

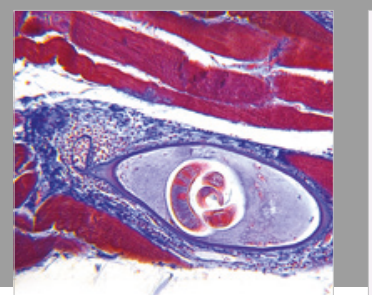

Gastroenterology Research and Practice

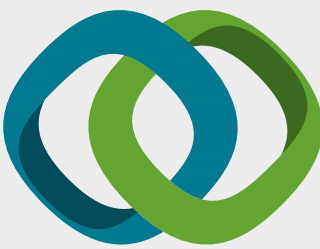

\section{Hindawi}

Submit your manuscripts at

www.hindawi.com
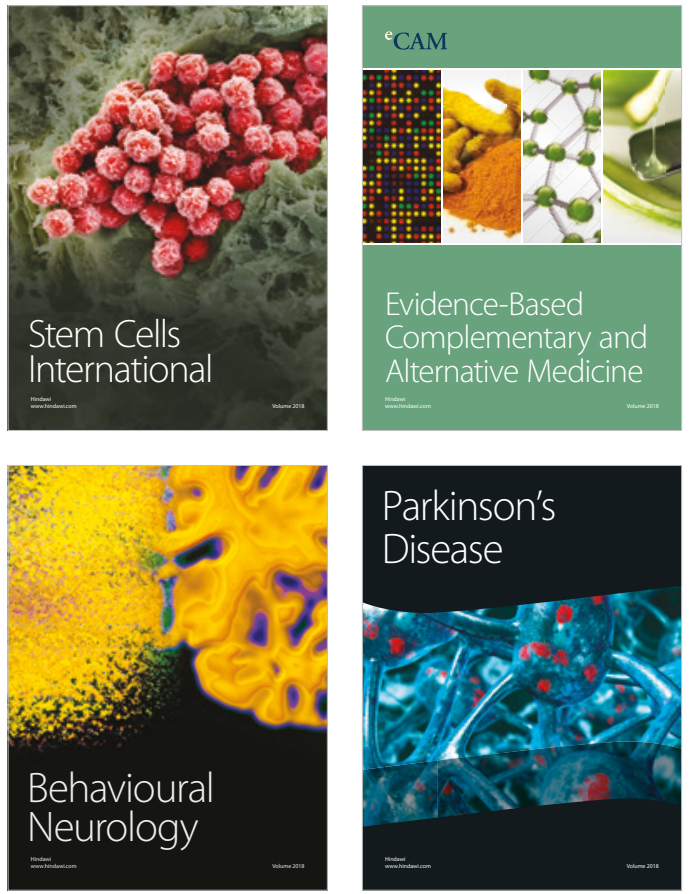

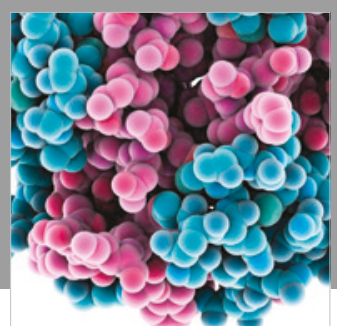

ournal of

Diabetes Research

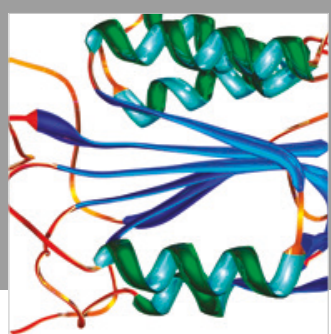

Disease Markers
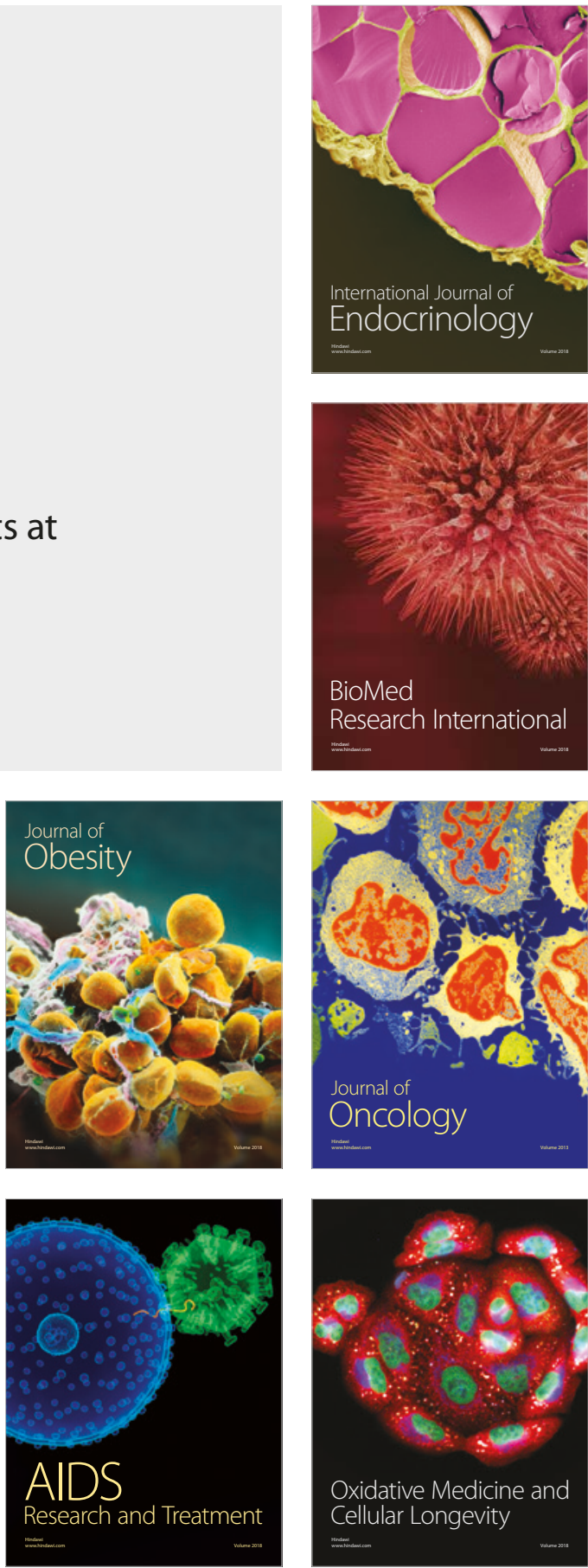